\title{
Optimum Design of Differential Unitary Space-Time Modulation
}

\author{
Mahdi Hajiaghayi and Chintha Tellambura, \\ Department of Electrical and Computer Engineering \\ University of Alberta \\ Edmonton, AB, Canada T6G 2V4 \\ Email: \{mahdih, chintha\}@ece.ualberta.ca
}

\begin{abstract}
In this paper, we propose an extended class of unitary signal constellations for differential unitary space-time modulation (DUSTM). We also derive an approximation of the upper bound on the symbol error probability (SEP) as a general criterion to find the optimum codes. This criterion is valid for both group or non-group constellations. For asymptotically high or low signal-to-noise ratio (SNR), signal-constellation parameters are usually determined based on the rank-and-determinant (diversity product) or the Euclidean distance (diversity sum) criterion. Since both these criterion are SNR-independent, the search results are not necessarily optimum parameters in medium to low SNRs. Thus instead of using diversity sum or product, we search for the constellation parameters to minimize the union-bound based criterion, taking into account the number of receive antenna and the operation SNR. Simulation results show that the constellations optimized for the union-bound based criterion outperform the previous codes resulting from rank-anddeterminant (diversity product) or Euclidean distance (diversity sum).
\end{abstract}

\section{INTRODUCTION}

Multiple-Input Multiple-Output (MIMO) systems increase the channel capacity significantly without the need to increase the bandwidth or transmit power [1]. The predicted MIMO capacity can be exploited by space-time (ST) codes [2], [3]. ST codes are typically designed for the case that the channel state information (CSI) is available at the receiver. However, it is difficult to ensure the availability of CSI when the channel changes rapidly. Moreover, channel estimation in some cases is either costly or impractical, if not impossible. Consequently, there are ST code designs that eliminate the need for the CSI at both the transmitter and receiver. Unitary space time modulation (USTM) [4] and differential USTM (DUSTM) [5] are two such schemes; they only require that the channel remains approximately constant over two block time intervals. There are two types of signal constellations (1) Group constellations (codes) [6], [7], every transmitted signal is still a codeword in the group code. (2) Non-Group Constellations, several of these codes have been discovered in [8]-[10]. It is remarked that the constellation design of nongroup codes is much easier than group codes.

In [11], it is argued that when the number of receive antennas is large, as a consequence low SNR at the receiver, the minimum Euclidean distance (Diversity Sum) among code words dominates the performance. Instead of maximizing diversity product or diversity sum as a design criterion, Wang et al. [11] optimized the codes such that the union bound on the SEP is minimized for particular number of receive and transmit antennas in specific operating SNR. However, it is shown that optimum codes with minimum union bound in a particular SNR outperforms the codes designed based on diversity product or diversity sum for a wide range of SNRs. They applied this new criterion to the cyclic group codes and presented some simulations. In this work, we first introduce an extended class of non-group constellation [10] and search for the optimum codes to minimize the union bound on the error probability.

Notation: $(\cdot)^{H}$ denotes conjugate transpose. The trace, determinant and the Frobenius norm of matrix $\mathbf{A}$ are trace $(\mathbf{A})$, $\operatorname{det}(\mathbf{A})$ and $\|\mathbf{A}\|_{F}^{2}=\operatorname{tr}\left(\mathbf{A} \mathbf{A}^{H}\right)$. A circularly complex Gaussian variable with mean $\mu$ and variance $\sigma^{2}$ is denoted by $z \sim \mathcal{C N}\left(\mu, \sigma^{2}\right)$. Matrix $I$ denotes the Identity matrix.

\section{System MOdel AND DUSTM}

We next present the channel model and summarize DUSTM. Consider a system with $M$ transmit and $N$ receive antennas. Let $h_{i, j}$ be the channel gain from the $i$-th transmit antenna to the $j$-th receive antenna. We assume all the channel gains to be independent and identically distributed (i.i.d.) $\mathcal{C N}(0,1)$ (Rayleigh fading) and remain constant for several symbol intervals. At time $t$, complex symbols $s_{t, i}$ are transmitted on antennas $i=1, \ldots, M$ and signal samples $y_{t, j}$ are received on antennas $j=1, \ldots, N$. The input-output relation is given by

$$
y_{t, j}=\sqrt{\rho} \sum_{i=1}^{M} h_{i, j} s_{t, i}+w_{t, j} \quad t=1, \ldots, T .
$$

The additive noise terms $v_{t, j}$ at time $t(t=1, \ldots, T)$ and on receiver antenna $j(j=1, \ldots, N)$ are i.i.d. $\mathcal{C N}(0,1) . \rho$ is the average signal-to-noise ratio (SNR) per receive antenna. All the samples in (1) can be rearranged in matrix form as

$$
\mathbf{Y}_{\tau}=\sqrt{\rho} \mathbf{S}_{\tau} \mathbf{H}_{\tau}+\mathbf{W}_{\tau}
$$

where $\tau$ is the block index, $\mathbf{Y}_{\tau}$ is the $T \times N$ complex received signal matrix, and $\mathbf{S}_{\tau}$ is the $T \times M$ complex transmitted signal matrix (block). Entries of $\mathbf{H}_{\tau}$ and $\mathbf{W}_{\tau}$ are i.i.d. $\mathcal{C N}(0,1)$. The sum of the average signal powers at each time instant is normalized to unity: $\operatorname{tr}\left(\mathbf{S}_{\tau} \mathbf{S}_{\tau}^{H}\right) / T=1$. Suppose a data sequence of integers $d_{1}, d_{2}, \ldots\left(d_{t} \in\{0, \ldots, L-1\}\right)$ is to be 
transmitted. Each $d_{t}$ is mapped to a matrix $\boldsymbol{\Phi}_{d_{t}}$ drawn from the constellation set of unitary matrices $\mathcal{S}=\left\{\boldsymbol{\Phi}_{l} \mid l=0, \ldots, L-\right.$ $1\}$. The positive integer $L \geq 2$ denotes the constellation size; and $L=2^{R M}$ where $R$ is the transmission data rate [bits/channel]. In differential systems, the signal matrix is $\mathbf{S}_{0}=\mathbf{I}$ is transmitted at the beginning. Thereafter, to send the data symbol $d_{\tau}, \boldsymbol{\Phi}_{d_{\tau}} \in \mathcal{S}$ is differentially encoded to give the following transmit signal matrix

$$
\mathbf{S}_{\tau}=\mathbf{\Phi}_{d_{\tau}} \mathbf{S}_{\tau-1} \quad \tau=1,2, \ldots
$$

Assuming that the channel remains constant for at least two block intervals (i.e., $\mathbf{H}_{\tau}=\mathbf{H}_{\tau-1}$ ), it is shown in [4] that the maximum-likelihood decision rule at the receiver is

$$
\widehat{d_{\tau}}=\underset{0 \leq l<L}{\operatorname{argmin}}\left\|\mathbf{Y}_{\tau}-\mathbf{\Phi}_{l} \mathbf{Y}_{\tau-1}\right\|_{F}^{2} .
$$

The exact pairwise probability of error (PEP) has been derived in [4], [7]. By assuming all messages are equally likely and $T=M$, we find that the exact PEP is given as [11]

$$
P_{l l^{\prime}}=p\left(\mathbf{\Phi}_{l} \longrightarrow \boldsymbol{\Phi}_{l^{\prime}}\right)=\frac{1}{\pi} \int_{0}^{\frac{\pi}{2}} \prod_{i=1}^{M}\left(1+\frac{\gamma \lambda_{i}}{4 \sin ^{2} \theta}\right)^{-N} d \theta
$$

where $\gamma=\frac{\rho^{2}}{1+2 \rho}$ and $\left\{\lambda_{i}\right\}$ is the $i$-th eigenvalue of the matrix $\Delta_{l l^{\prime}}=\left(\boldsymbol{\Phi}_{l}-\boldsymbol{\Phi}_{l^{\prime}}\right)\left(\boldsymbol{\Phi}_{l}-\boldsymbol{\Phi}_{l^{\prime}}\right)^{H}$. The Chernoff upper bound on the PEP is derived in [4]:

$$
P_{l l^{\prime}} \leq \frac{1}{2} \prod_{m=1}^{M}\left[1+\gamma \sigma_{m}\left(\mathbf{\Phi}_{l}-\mathbf{\Phi}_{l^{\prime}}\right)\right]^{-N}
$$

where $\sigma_{m}\left(\mathbf{\Phi}_{l}-\mathbf{\Phi}_{l^{\prime}}\right)$ is the $m$-th singular values of $\left(\mathbf{\Phi}_{l}-\mathbf{\Phi}_{l^{\prime}}\right)$. For asymptotically high SNR, it has been shown in [5] and [8] that the design criterion that minimizes the PEP is to maximize the diversity product:

$$
\begin{aligned}
\zeta_{l l^{\prime}} & =\frac{1}{2} \min _{l \neq l^{\prime}}\left|\operatorname{det}\left(\boldsymbol{\Phi}_{l}-\mathbf{\Phi}_{l^{\prime}}\right)\right|^{\frac{1}{M}} \\
& =\frac{1}{2} \min _{l \neq l^{\prime}}\left(\prod_{m=1}^{M} \sigma_{m}\left(\boldsymbol{\Phi}_{l}-\boldsymbol{\Phi}_{l^{\prime}}\right)\right)^{\frac{1}{M}} .
\end{aligned}
$$

On the other hand, for low SNR, the design criteria is to maximize the trace product which is called diversity sum [11]:

$$
\xi=\min _{l \neq l^{\prime}}\left\|\boldsymbol{\Phi}_{l}-\boldsymbol{\Phi}_{l^{\prime}}\right\|_{F}^{2}
$$

Recently, in [10], a new class of DUSTM constellations has been introduced; For $M$ transmit antennas, there are $M+1$ parameters that characterize this class. Typically, computer search is performed to find the optimal values of the parameters. An extended class of this constellation is proposed in next section.

\section{Proposed DUSTM Constellation} by

Consider a full-rotation matrix with rotation factors given

$$
R F_{M}(\underline{k} \theta)=\left(\begin{array}{ccc}
R F_{2}\left(k_{1} \theta\right) & \ldots & 0 \\
\vdots & \ddots & \\
0 & \ldots & R F_{2}\left(k_{\frac{M}{2}} \theta\right)
\end{array}\right)_{M \times M}
$$

where

$$
R F_{2}\left(k_{i} \theta\right)=\left(\begin{array}{cc}
\cos k_{i} \theta & \sin k_{i} \theta \\
-\sin k_{i} \theta & \cos k_{i} \theta
\end{array}\right)
$$

and $\underline{k}=\left\{k_{1}, k_{2}, \ldots, k_{\frac{M}{2}}\right\}$ is a set of different rotation factors. Our proposed DUSTM constellation $\mathcal{S}=\left\{\boldsymbol{\Phi}_{l} \mid l=0, \ldots, L-\right.$ $1\}$ consists of the following unitary matrices:

$$
\boldsymbol{\Phi}_{l}=\left(\begin{array}{ccc}
e^{j \theta_{L} \mu_{1}} & \ldots & 0 \\
\vdots & \ddots & \vdots \\
0 & \cdots & e^{j \theta_{L} \mu_{M}}
\end{array}\right)^{l} \cdot\left[R F_{M}\left(\underline{k} \theta_{L}\right)\right]^{l}
$$

where $l=0, \ldots, L-1$ and $\theta_{L}=\frac{2 \pi}{L}$. Clearly, this constellation is characterized by $\frac{3}{2} M$ parameters. When all $k_{i}$ 's are the same, our proposed constellation reduces to the constellation in [10]. Since our constellation has more parameters, we would expect better performance than previous designs; for example, our new constellation outperforms the previous codes in [10] and [4] in terms of the maximum diversity product.

By substituting the proposed constellation in (7), we can show that the diversity product is given by

$$
\begin{aligned}
\zeta_{l l^{\prime}}= & \frac{1}{2}\left|\operatorname{det}\left(\mathbf{\Phi}_{l}-\mathbf{\Phi}_{l^{\prime}}\right)\right|^{\frac{1}{M}} \\
= & \frac{1}{2} \prod_{i} \mid 1-\left(e^{j \Delta_{l} \Theta_{L} \mu_{i}}+e^{j \Delta_{l} \Theta_{L} \mu_{i+1}}\right) \cos k_{i} \Delta_{l} \Theta_{L} \\
& \quad+\left.e^{j \Delta_{l} \Theta_{L}\left(\mu_{i}+\mu_{i+1}\right)}\right|^{\frac{1}{M}}
\end{aligned}
$$

where $1 \leq i \leq M-1, i$ is odd and $\Delta_{l}=l^{\prime}-l$.

The integers $\mu_{1}, \ldots, \mu_{M} \in\{0, \ldots, L-1\}$ and $k_{1}, \ldots, k_{\frac{M}{2}} \in\{0, \ldots, L-1\}$ are parameters to be determined for the proposed constellation to yield the largest diversity product or the smallest union bound (Section IV). Since to the best of our knowledge there is no explicit solution to this minimax problem, we use exhaustive computer search. Candidates for the best set of $\underline{u}$ and $\underline{k}$ are exhaustively generated, tested for performance (minimum diversity product), and kept if they better the previously best candidate set. To reduce the search complexity, we can use several properties.

As (11) shows, $\zeta_{l l^{\prime}}$ depends only on the difference between $l$ and $l^{\prime}$. As a result, to determine the minimum of (11), there is no need to consider all values of $l$ and $l^{\prime}$ between 0 and $L-1$. Due to the symmetric structure of $\zeta$, it is sufficient to find the minimum of $\zeta_{0 l^{\prime}}$ over $l^{\prime}=1, \ldots, \frac{L}{2}$. One can further reduce the computational complexity by applying the following lemma.

Theorem 3.1: Due to $L$ is always even number, $\underline{\mu}$ and $\underline{k}$ should be in either of the below forms,

1) all $\mu_{i}$ 's are even numbers while all $k_{i}$ 's are odd numbers

2) all $\mu_{i}$ 's are odd integers number and all $k_{i}$ 's are even integer numbers.

Proof: See [10]. The same argument is applied here just by taking into account the different rotation angles instead of one angle in that specific structure.

As soon as the size of the constellation, $L$, increases above 16 , the search is time consuming, and, in some cases, may even 
be practically impossible. Since the computational complexity of finding optimum parameters grows exponentially with the increase of $M$ and $R$, following an idea from [10], we reduce the number of independent parameters in (10), resulting in the modified constellation

$$
\tilde{\boldsymbol{\Phi}}_{l}=\left(\begin{array}{ccc}
e^{j \theta_{L} \tilde{\mu}_{1}} & \ldots & 0 \\
\vdots & \ddots & \vdots \\
0 & \cdots & e^{j \theta_{L} \tilde{\mu}_{M}}
\end{array}\right)^{l} \cdot\left[R F_{M}\left(\underline{k} \theta_{L}\right)\right]^{l}
$$

where

$$
\tilde{\mu}_{k}= \begin{cases}\mu_{1}+2(k-1) & 1 \leq k \leq M / 2, \\ \mu_{2}+2 k-M-2 & M / 2<k \leq M .\end{cases}
$$

The first matrix in constellation (12) depends on just two parameters instead of $M$ parameters in constellation (10). Thus, the number of design parameters is reduced from $M+\frac{M}{2}$ to $\left(2+\frac{M}{2}\right)$ by this simplification. The modified constellation doesn't necessarily yield the maximum diversity product, but we find that it greatly reduces the search and its final result is quitely acceptable. The maximum diversity products of our proposed codes, codes in [10] and cyclic codes are presented in Table I. Our proposed constellation has equal or higher diversity product relative to the other constellations.

Thus far, we have considered the diversity product as the main design criterion, which is suitable for the high SNR regime. It is also argued in [11] that for a large number of receive antennas or low SNR scenarios, the Euclidean distance criterion (diversity sum) is suitable for DUSTM codes. Thus a question rises on the appropriateness of the design criteria at medium range of SNR. In the next section, we introduce a design criterion based on the union bound of the Symbol Error Probability (SEP).

\section{UPPER BOUND OF SEP AND NEW CRITERIA}

In differential modulation, the main target is usually to minimize the block error probability, not the symbol error probability (SEP). Because the code with optimum worst case in PEP do not necessarily yield the optimum performance in SEP, [11] minimized the union bound of SEP instead of minimizing PEP. But it has been done only for the group constellation. we consider our proposed non-group constellation.

Eq. (5) which is the exact PEP may be expressed in the form

$$
P_{l l^{\prime}}=\frac{1}{\pi} \int_{0}^{\frac{\pi}{2}} \frac{d \theta}{\operatorname{det}\left[I+\frac{\gamma}{4 \sin ^{2} \theta} \Delta_{l l^{\prime}}\right]^{N}}
$$

If $\sin \theta=t,(13)$ becomes

$$
P_{l l^{\prime}}=\frac{1}{2 \pi} \int_{-1}^{1} \frac{d t}{\operatorname{det}\left[I+\frac{\gamma}{4 t^{2}} \Delta_{l l^{\prime}}\right]^{N}} \frac{1}{\sqrt{1-t^{2}}}
$$

Using Chebyshev polynomials of first kind formula [12], the pairwise error probability may be expressed in the form

$$
P_{l l^{\prime}}=\frac{1}{2 n} \sum_{i=1}^{n} \frac{1}{\operatorname{det}\left[I+\frac{\gamma}{4 x_{i}^{2}} \Delta_{l l^{\prime}}\right]^{N}}+R_{n}
$$

\begin{tabular}{|c|c|c|c|c|}
\hline $\mathrm{M}$ & $\mathrm{L}$ & $\zeta($ proposed $)$ & $\zeta($ in [10]) & cyclic \\
\hline \multirow{3}{*}{6} & 16 & 0.5946 & 0.5946 & 0.5066 \\
\cline { 2 - 5 } & 32 & .5577 & .5069 & 0.448 \\
\hline \multirow{3}{*}{10} & 16 & 0.5946 & 0.5946 & 0.5623 \\
\cline { 2 - 5 } & 32 & .5655 & .5137 & 0.5131 \\
\hline
\end{tabular}

TABLE I

DIVERSITY PRODUCT OF THE OPTIMUM CODES WITH DIFFERENT CONSTELLATION SCHEME IN SIZE OF CONSTELLATION $L=16,32$

\begin{tabular}{|c|c|c|}
\hline Criterion & $\underline{\mu}$ & $\underline{k}$ \\
\hline Diversity Product maximizing & {$[31,33,35,19,21,23]$} & {$[28,28,28]$} \\
\hline Upper bound minimizing & {$[31,33,35,13,15,17]$} & {$[20,20,20]$} \\
\hline
\end{tabular}

TABLE II

OPTIMUM CODES BASED ON THE CONSTELLATION WITH ONE ROTATION FACTOR [10], $M=6, N=1, L=32$

where $x_{i}=\cos (2 i-1) \pi / 2 n$ and $R_{n}$ is a reminder term. If we take $n=9$, the reminder term will be sufficiently small to be ignored. In general, since the exact SEP is too complex to analyze, we have to turn to the union bound on the SEP. Based on equal priori probabilities assumption, the union bound would be

$$
P_{S E P} \leq P_{U B}=\frac{1}{L} \sum_{l=0}^{L-1} \sum_{l \neq l^{\prime}}^{L-1} P_{l l^{\prime}}
$$

With a good approximation $P_{U B}$ is equal to

$$
P_{U B}=\frac{1}{18 L} \sum_{l=0}^{L-1} \sum_{l \neq l^{\prime}}^{L-1} \sum_{i=1}^{9} \frac{1}{\operatorname{det}\left[I+\frac{\gamma}{4 x_{i}^{2}} \Delta_{l l^{\prime}}\right]^{N}} .
$$

Using MATLAB, the union bound in (18) is easy to compute. We also note from (18) that $P_{U B}$ depends on operation SNR and number of receive antennas as well as the number of transmit antenna and type of constellation.

Without loss of generality, we focus here on designing optimum DUST codes based on proposed constellation in section (III) and non-group constellation in [10]. The same procedure could be done for other types of constellations (group or non-group) as well. For a given set of $\mu$ and $\underline{k}$, the union upper bound is expressed by (18). Therefore, a good and reasonable design criteria could be to choose the sets $\mu$ and $\underline{k}$ that minimize the $P_{U B}$ for a particular predetermined $M, N$ and $\rho$. We resort to exhaustive computer searches for optimal set $\underline{\mu}$ and $\underline{k}$ since there is no explicit way to determine the optimum values. However, By recalling some restrictions in section (III) for choosing optimum parameters, we can reduce our search space by assuming them.

It is found from simulation that the codes with the minimum $P_{U B}$ at a specific SNR will also have near optimum union bound within a range of SNR. Therefore, for a medium range of SNR's for example in range from 4 to $12 \mathrm{~dB}$, which are of our interest here, the optimum code would be the same for this range of SNR. But, as we expect, for higher SNR's the codes that lead to minimum $P_{U B}$ would be different as well.

Some of the optimum codes based on two different criterions are presented in Table (II) and Table (III). To obtain those 


\begin{tabular}{|c|c|c|}
\hline Criterion & $\underline{\mu}$ & $\underline{k}$ \\
\hline Diversity Product maximizing & {$[31,33,35,29,31,33]$} & {$[20,20,30]$} \\
\hline Upper bound minimizing & {$[15,17,19,13,15,17]$} & {$[6,6,20]$} \\
\hline
\end{tabular}

TABLE III

OPTIMUM CODES BASED ON THE CONSTELLATION WITH THREE ROTATION FACTORS $M=6, N=1, L=32$

codes, we assumed $M=6$ transmit antennas and $N=1$ receive antenna with rate $R=\frac{5}{6}$.

\section{Simulation Results}

We simulated codes in Table II and Table III and codes in [5] and [11] and found that our proposed constellation performs better than the previously proposed constellations.

Fig. 1 compares the upper bound on block error rate performance of the codes represented in table (I). It also shows the union bound for other codes in [5] and [11]. As we mentioned in previous section, we assumed $M=6$ transmit antennas and $N=1$ receive antenna and the size of the constellation $L=32$. Our constellation and the proposed criterion can be applied in any number of receive and transmit antennas and for any data rate. We can see that union bound of our new code is smaller than that of the other codes.

Fig. 2 depicts the comparison between our new constellation with different rotation angles and fixed rotation angles in SEP from performance point of view. We have assumed a slow channel with Jakes' fading model with normalized fade rates of $f_{d} T_{s}=1.5 \times 10^{-3}$, where $f_{d}$ is the Doppler frequency and $T_{s}$ is a symbol duration. As we expect, the proposed constellation with different rotation angles (3 rotation angles for $M=6$ ) performs significantly. Furthermore, we observe that the union-bound based design generally has better performance than the design based on the diversity product or sum in both constellations.

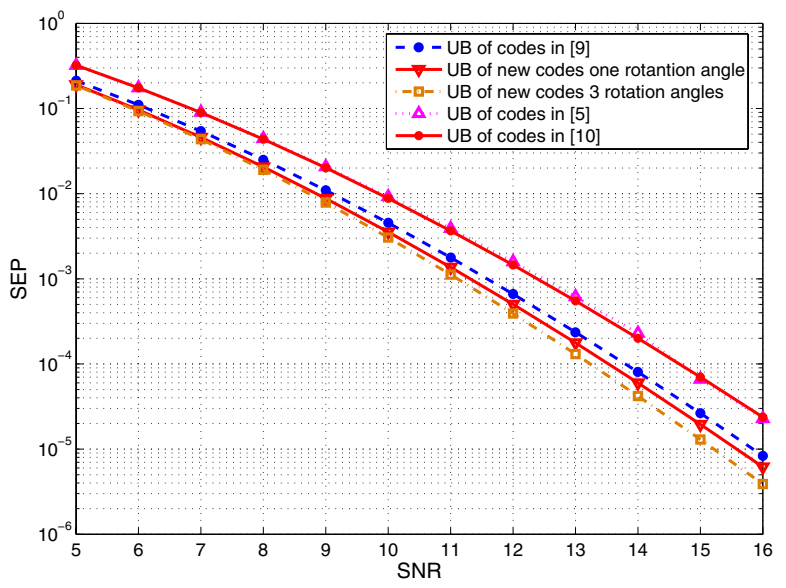

Fig. 1. Upper Bound on SEP for different codes, $M=6, N=1, L=32$.

\section{CONCLUSION}

In this paper, we have introduced an extended unitary signal constellation for DUSTM and presented a union-bound based criterion of optimal codes. Thus the parameters of the proposed constellation can be selected to optimize the diversity

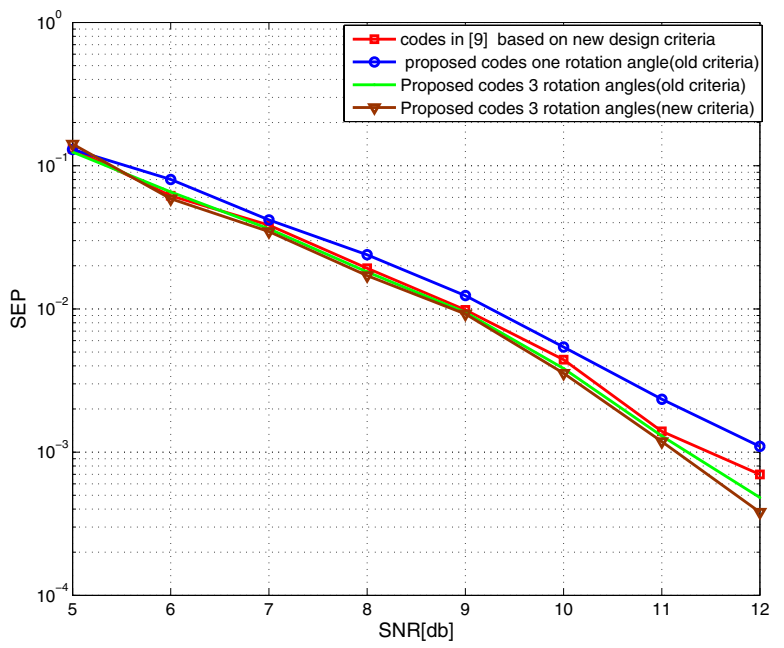

Fig. 2. The symbol Error Probability vs. SNR for 6 transmit antennas and 1 receive antenna and $L=32$.

product or the union bound. The optimum parameters to minimize the union bound are searched taking into account the number of receive antenna and the operational SNR. Simulation results showed that the resulting codes outperform the previous codes based on the maximization of rank-anddeterminant (diversity product criterion) or Euclidean distance (diversity sum criterion).

\section{REFERENCES}

[1] T. L. Marzetta and B. M. Hochwald, "Capacity of a mobile multipleantenna communication link in Rayleigh flat fading," IEEE Trans. Inform. Theory, vol. 45, pp. 139-157, Jan. 1999.

[2] E. Telatar, "Capacity of multi-antenna Gaussian channels," Euro. Trans. Telecommun., vol. 10, pp. 585 - 595, Nov. 1999.

[3] G. Foschini and M. Gans, "On limits of wireless communications in a fading environment when using multiple antennas," Wirel. Pers. Commun. (Netherlands), vol. 6, no. 3, pp. 311 - 335, Mar. 1998.

[4] B. Hochwald and T. Marzetta, "Unitary space-time modulation for multiple-antenna communications in Rayleigh flat fading," IEEE Trans. Inform. Theory, vol. 46, no. 2, pp. 543 - 564, Mar. 2000.

[5] B. Hochwald, T. Marzetta, T. Richardson, W. Sweldens, and R. Urbanke, "Systematic design of unitary space-time constellations," IEEE Trans. Inform. Theory, vol. 46, no. 6, pp. 1962 - 1973, Sept. 2000.

[6] A. Shokrollahi, B. Hassibi, B. Hochwald, and W. Sweldens, "Representation theory for high-rate multiple-antenna code design," IEEE Trans. Inform. Theory, vol. 47, no. 6, pp. 2335 - 2367, Sept. 2001.

[7] B. Hughes, "Differential space-time modulation," IEEE Transactions on Information Theory, vol. 46, no. 7, pp. 2567-2578, 2000.

[8] X. Liang and X. Xia, "Unitary signal constellations for differential spacetime modulation with two transmit antennas: Parametric codes, optimal designs, and bounds," IEEE Trans. Inform. Theory, vol. 48, pp. 2291 - 2322, Aug. 2002.

[9] T. P. Soh, C. S. Ng, and P. Y. Kam, "Improved signal constellations for differential unitary space-time modulations with more than two transmit antennas," IEEE Commun. Lett., vol. 9, no. 1, pp. 7 - 9, 2005.

[10] C. Shan, A. Nallanathan, and P. Y. Kam, "A new class of signal constellations for differential unitary space-time modulation (DUSTM)," IEEE Commun. Lett., vol. 8, no. 1, pp. 1- 3, 2004.

[11] J. Wang, M. Simon, and K. Yao, "On the optimum design of differential unitary space-time modulation," GLOBECOM '03. IEEE Global Telecommunications Conference (IEEE Cat. No.03CH37489), vol. 4, pp. $1968-72,2003$

[12] M. Abramowitz and I. A. Stegun, Handbook of Mathematical Functions with Formulas, Graphs, and Mathematical Tables. New York: Dover, 1972. 